Revue internationale P.M.E.

Économie et gestion de la petite et moyenne entreprise

\title{
Une expérience de parrainage : le cas de PME du secteur de l'énergie électrique
}

\section{Lise Préfontaine et France Pelletier}

Volume 11, numéro 1, 1998

URI : https://id.erudit.org/iderudit/1009036ar

DOI : https://doi.org/10.7202/1009036ar

Aller au sommaire du numéro

Éditeur(s)

Presses de l’Université du Québec

ISSN

0776-5436 (imprimé)

1918-9699 (numérique)

Découvrir la revue

Citer cet article

Préfontaine, L. \& Pelletier, F. (1998). Une expérience de parrainage : le cas de PME du secteur de l'énergie électrique. Revue internationale P.M.E., 11(1),

65-80. https://doi.org/10.7202/1009036ar

\section{Résumé de l'article}

La collaboration offre aux entreprises d'une même industrie une occasion intéressante de partager certaines ressources et expériences dans un climat d'entraide. Cette chance a été donnée à une cinquantaine d'entreprises, donneurs d'ordres et sous-traitants, oeuvrant dans le secteur de l'énergie électrique. Celles-ci ont eu la possibilité de participer à un projet de parrainage interfirmes dans lequel certaines agissaient en tant que parrains pour faciliter l'accréditation des autres (les filleuls) aux normes de qualité ISO 9000. Une enquête sur le terrain a été réalisée auprès des participants et des non-participants à ce projet afin de relever et de comparer certaines de leurs caractéristiques et motivations. Les résultats démontrent que les participants ne se différencient pas des non-participants en ce qui a trait à leur caractère innovateur et que les motifs qui incitent les entreprises à collaborer sont principalement d'ordre économique et stratégique. Déplus, l'expérience vécue laisse entrevoir toutes les difficultés, pour les organisations, d'adopter un changement d'esprit orienté vers la collaboration et non vers la compétition.
Ce document est protégé par la loi sur le droit d'auteur. L'utilisation des services d’Érudit (y compris la reproduction) est assujettie à sa politique d'utilisation que vous pouvez consulter en ligne.

https://apropos.erudit.org/fr/usagers/politique-dutilisation/ 


\title{
Une expérience de parrainage: le cas de PME du secteur de l'énergie électrique
}

\author{
Lise PRÉFONTAINE \\ Université du Québec à Montréal et CIRANO \\ France PELLETIER \\ Université du Québec à Montréal
}

MOTS CLÉS

\section{Parrainage - Collaboration interfirmes \\ Secteur de l'énergie électrique - Accréditation ISO 9000 \\ Motivations à collaborer - PME}

\begin{abstract}
RÉSUMÉ
La collaboration offre aux entreprises d'une même industrie une occasion intéressante de partager certaines ressources et expériences dans un climat d'entraide. Cette chance a été donnée à une cinquantaine d'entreprises, donneurs d'ordres et sous-traitants, œuvrant dans le secteur de l'énergie électrique. Celles-ci ont eu la possibilité de participer à un projet de parrainage interfirmes dans lequel certaines agissaient en tant que parrains pour faciliter l'accréditation des autres (les filleuls)
\end{abstract}

\section{LES AUTEURS}

Lise Préfontaine est professeure en technologies et systèmes d'information à l'École des sciences de la gestion de l'Université du Québec à Montréal et chercheure invitée au centre de recherche CIRANO. Ses recherches portent principalement sur la PME et sur les compétences organisationnelles qui soutiennent l'utilisation des technologies.

France Pelletier est étudiante diplômée du programme de MBA Recherche de l'Université du Québec à Montréal. Elle réalise des contrats de recherche dans le domaine de la gestion des ressources humaines pour l'entreprise privée et dans le cadre de recherches menées par des professeurs de l'UQAM et de l'École polytechnique de Montréal.

Adresse : Lise Préfontaine, C.P. 6192, succursale Centre-Ville, Montréal (Québec), H3C 4R2, Téléphone : (514) 987-3000, poste 4762, Télécopieur : (514) 987-3343,

Courriel: prefontaine.lise@uqam.ca 
aux normes de qualité ISO 9000. Une enquête sur le terrain a été réalisée auprès des participants et des non-participants à ce projet afin de relever et de comparer certaines de leurs caractéristiques et motivations. Les résultats démontrent que les participants ne se différencient pas des non-participants en ce qui a trait à leur caractère innovateur et que les motifs qui incitent les entreprises à collaborer sont principalement d'ordre économique et stratégique. De plus, l'expérience vécue laisse entrevoir toutes les difficultés, pour les organisations, d'adopter un changement d'esprit orienté vers la collaboration et non vers la compétition.

\begin{abstract}
Interfirm collaboration offers enterprises the opportunity to share certain resources and experiences and help each other. This opportunity has been given to fifty firms or so, contractors or subcontractors in the electrical sector. Firms had the possibility to participate in a sponsorship project where certain would act as sponsor or godfather to facilitate ISO 9000 accreditation of others (godchildren). An empirical study was performed with both participants and non participants in order to identify and compare their characteristics and motivations. Results show that participants to the sponsorship project do not differenciate themselves in regard to their innovative behavior. Both economic and strategic motives are invoked to motivate their desire to collaborate. The whole experience shows the difficulty for firms to collaborate when they have been competing with one another for so many years.
\end{abstract}

\title{
RESUMEN
}

La colaboración ofrece a las empresas de una misma industria una oportunidad de compartir recursos y experiencias en un clima de ayuda mutua. Esta oportunidad fue ofrecida a cerca de cincuenta empresas, contratantes y proveedores del sector energético. Las empresas podían tomar parte en un proyecto de apadrinamiento en el cual algunas empresas asumirían el papel de "padrino" para facilitar la acreditación de otras empresas " ahijados " a las normas de calidad ISO 9000. Con el fin de identificar las características y motivaciones de las empresas asumiendo uno u otro papel, se realizó una encuesta en la cual tomaron parte empresas participantes y no participantes. Los resultados muestran que no hay diferencia en cuanto al carácter innovador de las firmas participantes y no participantes y que los motivos que incitan las empresas a colaborar son de orden económico y estratégico. Además, la experiencia vivida permite entrever que las organizaciones tienen una gran dificultad a cambiar sus patrones de conducta y a adoptar un comportamiento de colaboración en vez de uno de competencia.

\section{Introduction}

À l'aube de l'an 2000, les entreprises prennent conscience qu'elles doivent adopter de nouvelles stratégies qui pourront les aider à s'adapter à un environnement changeant et de plus en plus compétitif. Deux stratégies connaissent actuellement une certaine ferveur dans les milieux d'affaires, à savoir le partenariat et les programmes de qualité totale. Un projet de parrainage regroupant ces deux stratégies a été 
proposé aux PME du secteur de l'énergie électrique par le ministère de l'Industrie, du Commerce, de la Science et de la Technologie et par Regroupement GrapÉlec, une association professio nnelle qui réunit les entreprises qui œuvrent dans la production, le transport et la distribution de l'énergie électrique au Québec. Ce projet vise à favoriser l'accréditation des entreprises de la grappe industrielle, et particulièrement les PME, aux normes de qualité ISO 9000.

L'objectif de cette recherche est de comparer les caractéristiques et les motivations entre participants et non-participants à ce projet de parrainage. Dans un premier temps, le concept de parrainage est comparé à des types de collaboration similaires pour ensuite être brièvement défini. Puis, une revue de la littérature permet de dresser le profil, en insistant sur les caractéristiques et les motivations, des entreprises qui privilégient la collaboration interfirmes. Une enquête sur le terrain, complétée par des entrevues auprès des principaux intervenants du milieu, est ensuite réalisée auprès des entreprises qui ont accepté ou refusé de participer au projet de parrainage mis de l'avant dans le secteur de l'énergie électrique dans le but d'établir une comparaison entre participants et non-participants. Les conclusions de la recherche mettent en lumière les différences de perception de la collaboration interfirmes de certains dirigeants d'entreprises de cette industrie et les implications de telles perceptions.

\section{Le parrainage comme forme de collaboration interfirmes}

L'utilisation du concept de parrainage dans un contexte d'affaires est relativement récent. Un survol de quelques formes de collaboration qui s'y apparentent est donc présenté afin de mieux faire ressortir les caractéristiques de cette forme de collaboration interfirmes.

Plusieurs domaines de recherche ont proposé des théories sur la collaboration entre entreprises (Ring Smith et Van de Ven, 1994). À titre d'exemple, la littérature sur le mentorat s'intéresse au transfert de connaissances entre individus d'une même entreprise et introduit le concept de mentor, lequel comporte une relation d'aide ou de soutien entre le mentor, ou guide, et son protégé. La relation entre le parrain et le filleul s'apparente à ce type, bien que certaines limites s'imposent. D'abord, le mentorat implique un transfert de connaissances entre individus plutôt qu'entre entreprises. De plus, en règle générale, mentors et protégés sont assignés les uns aux autres alors que dans le cas des parrains et des filleuls, il s'agit d'un libre choix de partenaires.

L'étalonnage ou benchmarking consiste en la comparaison que réalisent deux ou plusieurs entreprises de leurs processus d'affaires où l'une sert de modèle ou d'étalon à l'autre qui cherche à s'améliorer. Trop souvent, l'étalonnage s'établira sans le consentement explicite de l'entreprise servant d'étalon qui a développé, au prix de nombreux efforts, d'excellentes pratiques de gestion ou de production. Au 
contraire, le parrainage s'établit habituellement dans un climat de confiance et d'entraide. D'ailleurs, dans de nombreux cas, entreprises parraines et filleules agissent déjà comme partenaires commerciaux dans une relation de donneur d'ordres et sous-traitant. Le parrain qui aide une entreprise sous-traitante à obtenir une accréditation ISO 9000 améliore par le même fait la qualité de certains composants et en retire donc un avantage évident.

La littérature sur la collaboration interfirmes propose une abondance d'écrits touchant les différentes formes d'alliances, la nature de la collaboration et son ampleur (Gray et Wood, 1991). Ces différents liens interfirmes vont de la soustraitance de capacité ou de spécialité à des liens plus resserrés dans le cas de la coentreprise. Ainsi, Guilhon (1993) identifie la firme-réseau, mode d'organisation grâce auquel des ressources sont mises en commun pour réaliser un projet. Pour Mody (1993), une alliance serait une organisation flexible qui permet aux firmes ayant des forces complémentaires d'expérimenter de nouvelles stratégies de nature technologique, organisationnelle ou de marketing. Ce dernier différencie les alliances horizontales (entre compétiteurs) des alliances verticales qui réfèrent aux relations entre partenaires dans la chaîne de valeur, par exemple, le lien clientfournisseur. Alors que Westley et Vredenburg (1991) introduisent la notion de résolution de problèmes comme objet premier de la collaboration, Ring Smith et Van de Ven (1994) considèrent le caractère social de la collaboration et incluent la volonté des individus à mettre sur pied des mécanismes d'action collective. Ce sont Gray et Wood (1991) qui résument le mieux toutes ces dimensions en définissant la collaboration comme un effort organisationnel pour régler des problèmes complexes par une action bilatérale.

Mais qu'est-ce que le parrainage ? Selon la Société québécoise de développement de la main-d'œuvre, le parrainage consiste en un jumelage entre dirigeants d'entreprises où l' un agit à titre de personne-ressource pour soutenir l'autre dans une démarche de renforcement de ses habiletés à gérer son entreprise (SQDM, 1994). Le soutien offert prend la forme d'informations, de conseils et d'un éclairage concernant des questions fonctionnelles ou stratégiques. Cette définition recoupe les divers types de collaboration vus précédemment : elle inclut la notion d'aide du mentorat, celle de modèle à imiter de l'étalonnage et celle de collaboration interfirmes, puisque le concept de parrainage implique une alliance de collaboration.

\section{Caractéristiques des entreprises qui collaborent}

Toute entreprise est appelée à prendre des décisions qui se résument généralement à l'une des quatre options suivantes : faire, ne pas faire, faire faire et faire ensemble (Poulin, Montreuil et Gauvin, 1994). Le parrainage s'inscrit dans cette dernière option stratégique qui s'offre à l'entreprise qui choisit délibérément la collaboration comme moyen d'améliorer sa performance, de réduire l'incertitude entourant son 
environnement compétitif, de créer et de maintenir un réseau d'affaires et de prendre sa place dans l'industrie et dans la société en général (Ibid.).

L'entreprise qui recherche le parrainage se distingue d'abord par sa volonté d'établir un esprit et une relation de réciprocité avec ses partenaires (Julien, 1994). La culture organisationnelle, c'est-à-dire les valeurs, les prémisses guidant décisions et actions et les croyances (Allaire et Firsirotu, 1993), devraient donc refléter ce désir de collaborer. Pour Kanter (1983), un climat ouvert, souple et confiant constitue le principal atout de l'entreprise de type organique par opposition à une entreprise mécanique qui privilégie le contrôle et une structure plus rigide. Dans une analyse des relations entre client et fournisseur, Kumar (1996) insiste sur le fait que les collaborateurs se caractérisent par une culture, des personnes, des systèmes de gestion et des attitudes axées sur la confiance et l'ouverture plutôt que sur le pouvoir et le contrôle.

Cette ouverture se traduira vraisemblablement par l'utilisation de mécanismes de gestion dits « innovateurs ». Ainsi, ce désir de collaborer encouragera à l' interne la mise en place de mécanismes de décision participatifs (Birley et Westhead, 1990 ; Nebenhaus, 1990). L'entreprise qui valorise le partenariat avec ses clients et fournisseurs soignera également les relations avec ses propres employés et misera sur leur formation pour s'assurer d'une main-d'œuvre motivée et compétente (Hornsby et Kuratko, 1990 ; Naro, 1990, Uzan, 1990). L'adoption de programmes de qualité et l'accréditation ISO 9000 constituent, en effet, des changements organisationnels importants dont le succès repose sur la participation des employés et sur leurs compétences (Gagné et Lefèvre, 1993).

Sur le plan stratégique, Ettlie (1983) démontre que les entreprises innovatrices adoptent une stratégie manufacturière bien définie et ciblée sur la qualité, la réduction des coûts et la diversité. Elles cherchent constamment à réduire l'écart entre les objectifs qu'elles poursuivent et ceux que, dans les faits, elles atteignent. Les entreprises qui visent plus particulièrement la qualité (dont l'accréditation des processus de production ISO 9000 en est un aspect) chercheront les moyens les plus efficaces et les plus efficients pour obtenir cette accréditation. La création d'un réseau d'affaires constitue une avenue prometteuse (Préfontaine, Lefebvre et Lefebvre, 1995). Ettlie et Penner-Hahn (1990) soulignent également que l'établissement de liens avec les divers partenaires de la chaîne de valeur contribue à donner à l'entreprise plus de flexibilité et une performance accrue en lui assurant l'accès à des ressources complémentaires.

\section{Les motivations à collaborer}

Plusieurs types de motivations peuvent pousser les entreprises à s'engager dans une relation de collaboration. Le tableau 1 présente les principales catégories de motifs qui incitent les entreprises à collaborer ainsi que quelques auteurs qui ont traité de ces catégories. 


\section{TABLEAU 1}

\section{Catégorie de motifs pour solliciter et justification théorique}

Catégories de motifs

Motifs purement économiques

- Profitabilité

- Économies d'échelle

- Réduction des coûts de transactions

Motifs stratégiques

- Interdépendance entre partenaires

- Réduction de l'incertitude et partage du risque

- Transfert de connaissances et apprentissage

- Accès à des ressources complémentaires

Motifs réglementaires ou d'obligation

- Réglementation gouvernementale

- Obligation de l'industrie

- Interdépendance entre partenaires

Motifs sociaux et culturels

- Développement et maintien de réseaux d'affaires

- Plaisir de collaborer et confiance mutuelle

- Valorisation de l'entraide et de la collaboration

- Développement et maintien d'une image de marque
Justification théorique

Gray et Wood, 1991

Masse, 1992

Williamson, 1981

Ring Smith et Van de Ven, 1994

Burgers, Hill et Chan, 1993

Mody, 1993

Browning, Beyer et Shetler, 1995

Gray et Wood, 1991

Browning, Beyer et Shetler, 1995

Ring Smith et Van de Ven, 1994

Ring Smith et Van de Ven, 1994

Browning, Beyer et Shetler, 1995

Masse, 1992

Sharman, Gray et Yan, 1991

La théorie de l'économie des organisations met l'accent sur les motifs de nature économique qui encouragent les entreprises à maximiser leur efficacité en réduisant leurs coûts de transactions (Williamson, 1981 ; Gray et Wood, 1991). Les entreprises recherchent alors des bénéfices monétaires sous forme d'économies d'échelle ou de réduction de coûts (Masse, 1992).

La recherche d'une plus grande profitabilité passe très souvent par la recherche d'avantages stratégiques qui nécessitent l'accès ou l'acquisition de ressources complémentaires de nature technologique, commerciale ou informationnelle (Ring Smith et Van de Ven, 1994). Le partenariat favorise alors une réduction de l'incertitude environnementale en réduisant la concurrence ou en favorisant l'accès à de nouveaux marchés (Burgers, Hill et Chan, 1993). La mise en commun de ressources encourage le transfert de connaissances et l'apprentissage (Mody, 1993). Les entreprises partenaires partagent alors leurs expériences sur les problèmes de production ou de gestion et bénéficient des solutions innovatrices développées par l'un ou l'autre des partenaires. Ce fait est confirmé par une étude portant sur la coopération dans l'industrie des semi-conducteurs et réalisée par Browning, Beyer et Shetler (1995). Les auteurs concluent que la mise en commun de ressources et la réponse à la menace des concurrents sont deux motifs conventionnels poussant les entreprises à s'engager dans des activités de coopération. Selon les auteurs, cette coopération implique non seulement la coordination d'activités communes, mais également le partage de bénéfices. 
Dans certains cas, des réglementations créent une obligation qui force les entreprises à collaborer. Dans le contexte particulier du secteur de l'électricité, de fortes pressions s'exercent sur l'industrie et ses grands donneurs d'ordres : pour devenir fournisseurs de classe mondiale, ceux-ci doivent adhérer aux normes internationales de qualité ISO 9000. Hydro-Québec a donc décrété que tous ses fournisseurs devraient être accrédités pour la fin de 1997. Bien que les entreprises soient libres quant aux moyens à utiliser pour mettre en place leur programme de qualité totale, ces exigences du marché ne sont pas sans créer un sentiment d'obligation et d'urgence. L'interdépendance des partenaires crée un tel sentiment d'obligation puisque accéder aux volontés d'un client est souvent le seul moyen de le conserver (Ring Smith et Van de Ven, 1994 ; Browning, Beyer et Shetler, 1995). Hart et Saunders (1997) ont noté que des partenaires plus puissants exercent parfois un pouvoir coercitif pour forcer l'adoption de technologies comme l'échange de données informatisé (ÉDI).

Les motifs poussant les entreprises à collaborer ne sont pas qu'économiques. Sharman, Gray et Yan (1991) ont identifié des motifs sociaux et culturels, tels que le besoin de démontrer sa bonne volonté, le désir de rehausser l'image de l'industrie ou de développer une image de marque, qui incitent nombre d'entreprises à s'engager dans des liens de partenariat. Ring Smith et Van de Ven (1994) vont dans le même sens lorsqu'ils affirment que l'appartenance à un groupe favorise le sentiment d'identité et d'inclusion autant chez les humains que dans les entreprises. Finalement, la collaboration peut créer un sentiment de satisfaction issu de la coréalisation d'un projet avec des partenaires dans un climat de confiance et de respect mutuel (Browning, Beyer et Shetler, 1995 ; Masse, 1992).

Cette réflexion sur la collaboration entre organisations a permis de relever quelques caractéristiques des entreprises susceptibles d'établir des liens de partenariat avec d'autres entreprises : il s'agit d'entreprises innovatrices qui sont à l'affût de nouveaux savoirs et savoir-faire. Bien que ces entreprises soient principalement animées par des motifs économiques et stratégiques, elles recherchent certains avantages intangibles tels que la reconnaissance par les pairs, le sentiment d'appartenance à un réseau d'affaires et la satisfaction d'avoir aidé et participé à l'atteinte d'objectifs communs. L'étude sur le terrain réalisée dans le cadre de ce projet de recherche vise à confirmer - ou à infirmer - si les entreprises participantes au projet de parrainage dans le but de faciliter l'accréditation aux normes ISO 9000 se distinguent des entreprises non participantes en ce qui a trait aux caractéristiques et motivations suivantes :

- au regard des caractéristiques organisationnelles: une culture de type organique, une stratégie manufacturière innovatrice, des mécanismes de décision participatifs et des pratiques de gestion des ressources humaines et de formation avant-gardistes ; 
- au regard des motivations à collaborer: l'accent sur les motifs économiques et stratégiques qui laissent présumer d'une plus grande proactivité à l'égard de l'environnement d'affaires, plutôt que sur les motifs de nature obligatoires, culturels ou sociaux.

\section{Méthodologie de recherche}

Une enquête sur le terrain a été réalisée auprès d'un nombre restreint d'entreprises membres de Regroupement GrapÉlec, association qui réunit les entreprises grandes ou petites qui œuvrent dans le secteur de la production, de la distribution et du transport de l'énergie électrique. Les entreprises avaient préalablement été identifiées par l'association comme susceptibles de participer à un projet de parrainage dans le but de faciliter l'implantation des normes ISO 9000. En tout, 51 firmes avaient été pressenties pour agir à titre de parrains (9) ou de filleuls (42). Toutes ces entreprises ont également été invitées à participer à cette étude. Quatre questionnaires prétestés et distincts, mais comportant certaines sections similaires, ont été envoyés aux 30 parrains et filleuls ayant accepté de participer au projet de parrainage ainsi qu'aux 21 autres qui ont refusé de le faire. Le taux de réponse global se situe à 56,9\% et la répartition des entreprises par sous-groupes de participants et de nonparticipants au parrainage est présentée au tableau 2. Les répondants occupent divers postes tous reliés à la gestion de la qualité, soit directeurs de programmes d'assurance qualité ou de contrôle de la qualité, directeurs ou responsables de la production ou même p.-d.g. de l'entreprise. Les données quantitatives recueillies ont été complétées par des informations qualitatives obtenues par des entrevues auprès de quelques intervenants actifs dans ce secteur d'activité.

TABleau 2

Taux de réponse par sous-groupes de participants et de non-participants au parrainage

\begin{tabular}{lccccc}
\hline & $\begin{array}{c}\text { Parrains } \\
\text { participants } \\
\text { au parrainage }\end{array}$ & $\begin{array}{c}\text { Filleuls } \\
\text { participants } \\
\text { au parrainage }\end{array}$ & $\begin{array}{c}\text { Parrains } \\
\text { non } \\
\text { participants } \\
\text { au parrainage }\end{array}$ & $\begin{array}{c}\text { Filleuls } \\
\text { non } \\
\text { participants } \\
\text { au parrainage }\end{array}$ & Total \\
\hline Population & 2 & 28 & 7 & 14 & 51 \\
Nombre de répondants & 2 & 17 & 3 & 7 & 29 \\
Taux de réponse & $100 \%$ & $61 \%$ & $43 \%$ & $50 \%$ & $57 \%$ \\
\hline
\end{tabular}

Les principales caractéristiques des entreprises répondantes ont été évaluées à l'aide de construits préalablement testés et utilisés dans d'autres recherches. Le tableau 3 fournit la liste de ces construits, le nombre de points qui les composent, le coefficient de fidélité ainsi que les noms des auteurs qui les ont développés ou 
inspirés. Les motivations ont été évaluées à partir d'une liste de 52 énoncés issus des recherches des auteurs cités au tableau 1 et regroupés selon les quatre catégories de motifs suivantes : $i$ ) économiques, $i i$ ) stratégiques, $i i i$ ) réglementaires ou d'obligation et $i v$ ) sociaux et culturels. Les répondants ont indiqué, sur une échelle à sept points d'ancrage, l'importance relative qu'ils accordaient à chacun des motifs dans leur décision de participer au projet de parrainage ou dans la décision qu'ils pourraient prendre dans le cas des non-participants.

TABleau 3

Mesures utilisées, nombre d'énoncés, coefficient de fidélité et auteurs qui les ont développées ou inspirées

\begin{tabular}{lccl}
\hline \multicolumn{1}{c}{ Mesure } & $\begin{array}{c}\text { Nombre } \\
\text { d'énoncés }\end{array}$ & $\begin{array}{c}\text { Coefficient } \\
\text { de fidélité }\end{array}$ & Auteurs \\
\hline $\begin{array}{l}\text { Mécanismes de formation } \\
\text { Mécanismes de gestion }\end{array}$ & 5 & 0,82 & Adapté de Rosanvallon, 1990 \\
des ressources humaines & 8 & 0,80 & Adapté de Hornsby et Kuratko, 1990 \\
Mécanismes de décision & 6 & 0,89 & Adapté de Birley et Westhead, 1990 \\
Organicité & 7 & 0,73 & Covin et Slevin, 1990 \\
Stratégie manufacturière & 9 & 0,91 & Ettlie et Penner-Hahn, 1990 \\
\hline
\end{tabular}

1. Mesuré par l'alpha de Cronbach avec $n$ variant entre 27 et 29 selon le construit considéré.

Compte tenu du nombre restreint de répondants, les statistiques compilées se résument à des distributions de fréquences, à des moyennes et écarts types et à quelques tests de comparaison de moyennes.

\section{Caractéristiques des participants et des non-participants}

Les 29 entreprises qui ont retourné un questionnaire emploient 212 personnes en moyenne et sont en affaires depuis près de 50 ans. En réalité, les entreprises filleules sont des PME dont le nombre moyen d'employés est de 63 alors que ce nombre atteint 320 pour les parrains qui agissent à titre de donneurs d'ordres. La majorité des entreprises en sont à leur première expérience de parrainage, à l'exception de quelques parrains ayant déjà expérimenté un tel type de collaboration.

L'examen du tableau 4 permet de visualiser les différences entre participants et non-participants au projet de parrainage quant aux caractéristiques organisationnelles retenues comme susceptibles d'affecter le choix de l'entreprise de participer ou non à ce projet. Les résultats pour chacun des sous-groupes parrains et filleuls sont également fournis. La première comparaison effectuée concerne le degré d'organicité de la culture organisationnelle qui s'avère légèrement plus élevé chez les participants avec une différence de 0,22. 
TABLEAU 4

Caractéristiques organisationnelles des participants et des non-participants au projet de parrainage

\begin{tabular}{lcccccc}
\hline & \multicolumn{3}{c}{ Participants } & \multicolumn{3}{c}{$\begin{array}{c}\text { Non-participants } \\
\text { au parrainage }\end{array}$} \\
\hline Caractéristiques des $_{\text {entreprises }}{ }^{1}$ & $\begin{array}{c}\text { Parrains } \\
\boldsymbol{n}=\mathbf{2}\end{array}$ & $\begin{array}{c}\text { Filleuls } \\
\boldsymbol{n}=\mathbf{1 7}\end{array}$ & $\begin{array}{c}\text { Moyenne } \\
\boldsymbol{n}=\mathbf{1 9}\end{array}$ & $\begin{array}{c}\text { Parrains } \\
\boldsymbol{n}=\mathbf{3}\end{array}$ & $\begin{array}{c}\text { Filleuls } \\
\boldsymbol{n}=\mathbf{7}\end{array}$ & $\begin{array}{c}\text { Moyenne } \\
\boldsymbol{n}=\mathbf{1 0}\end{array}$ \\
\hline Organicité & 5,11 & 4,90 & $\mathbf{4 , 9 2}$ & 4,81 & 4,65 & $\mathbf{4 , 7 0}$ \\
Stratégie manufacturière & 5,83 & 4,01 & $\mathbf{4 , 2 0}$ & 5,30 & 4,11 & $\mathbf{4 , 4 7}$ \\
Mécanismes de décision & 5,83 & 4,12 & $\mathbf{4 , 3 0}$ & 5,56 & 3,71 & $\mathbf{4 , 2 7}$ \\
Mécanismes de GRH & 5,19 & 3,85 & $\mathbf{3 , 9 9}$ & 6,29 & 3,98 & $\mathbf{4 , 6 8}$ \\
Mécanismes de formation & 4,80 & 3,78 & $\mathbf{3 , 8 8}$ & 6,00 & 3,69 & $\mathbf{4 , 3 8}$ \\
\hline
\end{tabular}

1. Mesurées sur une échelle de Likert à sept points d'ancrage où 1 correspond à peu important et 7 , à très important.

On constate que les participants se différencient par une culture légèrement plus organique qui laisse entrevoir un climat organisationnel plus ouvert, souple et confiant. Un point est surprenant, soit le fait que dans les deux cas, le degré d'organicité des parrains dépasse celui des filleuls. Diverses études, dont celle de Acs et Audretsch (1989), soulignent pourtant le caractère flexible et la grande capacité d'adaptation des PME en comparaison des entreprises de plus grande taille. Peut-être sommes-nous en présence d'entreprises parraines qui privilégient la participation et la collaboration à un haut niveau et qui démontrent ainsi une grande souplesse et une ouverture d'esprit peu commune.

La stratégie manufacturière adoptée par les participants ressort comme légèrement moins innovatrice, autant en ce qui concerne la qualité, la réduction des coûts ou la diversité, que celle des non-participants. Nous sommes en présence de parrains dont la stratégie est bien définie $(5,83$ et 5,30 respectivement pour les participants et les non-participants) alors que cette stratégie apparaît moins bien précisée chez les entreprises filleules (4,01 et 4,11). Ces résultats ne concordent pas avec ceux obtenus par Lefebvre, Lefebvre et Préfontaine (1993) qui démontraient que les PME manufacturières innovatrices se caractérisent par une stratégie manufacturière axée principalement sur la qualité et la diversité, à moins que les participants à ce projet ne correspondent pas au profil d'entreprises innovatrices. On pourrait en effet penser que les entreprises filleules les plus innovatrices de ce secteur industriel ont déjà adhéré à un programme de qualité et sont en possession d'une accréditation ISO 9000 de sorte que celles qui ont été pressenties pour participer à ce projet sont en réalité des «adopteurs tardifs » selon la typologie établie par Rogers (1983). 
Les différences dans l'importance accordée aux diverses pratiques de gestion ne correspondent pas aux sens prévus. Les participants ne diffèrent pas des nonparticipants quant aux mécanismes de gestion participative mis en place. Mais ils ont toutefois adopté des mécanismes de gestion des ressources humaines et de formation moins avant-gardistes que ne l'ont fait les non-participants. Dans le cas particulier des mécanismes de gestion des ressources humaines, la différence entre participants et non-participants s'avère assez importante, soit de 0,69 . Autant chez les participants que chez les non-participants, les parrains devancent les filleuls, ce qui reflète une structuration et une formalisation plus poussées des mécanismes de gestion adoptés. Dans les petites entreprises, la mise en place de pratiques formelles de gestion est particulièrement difficile, compte tenu de la rareté des ressources humaines et financières (Rothwell, 1991). La différence observée entre participants et non-participants pourrait s'expliquer, du moins en partie, par le fait que les non-participants considèrent qu'ils détiennent les ressources internes appropriées pour implanter un tel programme alors que les entreprises participantes, conscientes de leurs ressources limitées, entrevoient la possibilité de profiter d'un soutien complémentaire externe grâce au parrainage.

\section{Les motivations invoquées pour collaborer}

Dans le but de mieux comprendre le comportement de collaboration des entreprises, les motivations qui ont de fait conduit les entreprises à participer et celles qui pourraient susciter l'adhésion des non-participants au parrainage ont été inventoriées. Ces résultats sont présentés au tableau 5. Les participants, parrains et filleuls, invoquent d'abord et avant tout des motifs d'ordre stratégique pour justifier leur choix. Les autres catégories de motifs mentionnées sont, par ordre d'importance, les motifs sociaux et culturels suivis des motifs purement économiques. Viennent en dernier lieu les motifs réglementaires ou d'obligation. Quant aux non-participants, ils considèrent que certains motifs pourraient les inciter à s'impliquer dans un tel projet dans un avenir prochain : d'abord, des motifs économiques et stratégiques et ensuite, des motifs sociaux et culturels ainsi que réglementaires. On constate encore une fois certaines différences entre les sous-groupes : ainsi, les parrains participants se sont sentis obligés de le faire, peut-être parce que ces deux parrains sont perçus comme des leaders de l'industrie et qu'ils sont conscients de l'influence qu'ils exercent et de l'importance d'une telle accréditation pour accéder aux marchés internationaux.

Pour l'ensemble des répondants à cette enquête, l'importance des motifs stratégiques et économiques ressort de façon évidente puisque pour les 29 entreprises, l'importance relative de ces deux catégories atteint 5,2 et 4,7 respectivement. Les motifs sociaux et culturels suivent avec une moyenne de 4,6 alors que les motifs réglementaires et d'obligation sont classés au dernier rang avec une moyenne 


\section{TABleau 5}

Motifs invoqués par les participants et les non-participants au projet de parrainage

\begin{tabular}{lcccccc}
\hline & \multicolumn{3}{c}{$\begin{array}{c}\text { Participants } \\
\text { au parrainage }\end{array}$} & \multicolumn{3}{c}{$\begin{array}{c}\text { Non-participants } \\
\text { au parrainage }\end{array}$} \\
\hline $\begin{array}{l}\text { Motifs invoqués pour } \\
\text { expliquer leur choix }\end{array}$ & $\begin{array}{c}\text { Parrains } \\
\boldsymbol{n}=\mathbf{2}\end{array}$ & $\begin{array}{c}\text { Filleuls } \\
\boldsymbol{n}=\mathbf{1 7}\end{array}$ & $\begin{array}{c}\text { Moyenne } \\
\boldsymbol{n}=\mathbf{1 9}\end{array}$ & $\begin{array}{c}\text { Parrains } \\
\boldsymbol{n}=\mathbf{1}\end{array}$ & $\begin{array}{c}\text { Filleuls } \\
\boldsymbol{n}=\mathbf{7}\end{array}$ & $\begin{array}{c}\text { Moyenne } \\
\boldsymbol{n}=\mathbf{1 0}\end{array}$ \\
\hline $\begin{array}{l}\text { Motifs purement } \\
\text { économiques }\end{array}$ & & & & & & \\
Motifs stratégiques & $5,33(4)^{\mathrm{ii}}$ & $4,46(3)$ & $\mathbf{4 , 4 5}(\mathbf{3})$ & 5,56 & 5,12 & $\mathbf{5 , 1 8 ( 1 )}$ \\
$\begin{array}{l}\text { Motifs réglementaires } \\
\text { ou d'obligation }\end{array}$ & $5,09(1)$ & $5,42(1)$ & $\mathbf{5 , 3 9}(\mathbf{1})$ & 5,85 & 4,67 & $\mathbf{4 , 8 3}(\mathbf{2})$ \\
Motifs sociaux et culturels & $4,75(2)$ & $4,22(4)$ & $\mathbf{4 , 2 8 ( 4 )}$ & 5,50 & 4,12 & $\mathbf{4 , 2 9 ( 4 )}$ \\
\hline
\end{tabular}

i Mesurés par 58 énoncés sur une échelle de Likert à sept points d'ancrage, où 1 correspond à peu important et 7 , à très important, regroupés en quatre catégories.

ii Rang selon l'importance de la catégorie de motifs invoqués.

de 4,3. Malgré les diverses pressions qui s'exercent sur l'industrie électrique, ces entreprises considèrent que le parrainage améliorera leur compétitivité et leur profitabilité et accordent moins d'importance à la nécessité ou à l'obligation dans leur décision d'adhérer à ce projet de collaboration interfirmes.

\section{Rétrospective sur l'expérience de parrainage}

Au moment où cette expérience se poursuit et que plusieurs autres PME ont sollicité le soutien de Regroupement GrapÉlec pour l'obtention d'une accréditation aux normes internationales ISO 9000, il est intéressant de faire le point sur ce début d'expérience de collaboration interfirmes, sur le profil des entreprises collaboratrices et sur celui des non-collaboratrices. Les leçons tirées de cette expérience dans l'industrie électrique, une industrie mature et grandement affectée par la morosité du climat économique, pourront éventuellement inspirer d'autres secteurs industriels qui doivent affronter une concurrence mondiale qui ne cesse de s'accroître.

Même si de nombreuses études démontrent les avantages de la collaboration, en convaincre les entreprises n'est pas chose facile. Des firmes habituées à considérer leurs pairs comme des compétiteurs sur le marché local éprouvent des difficultés à percevoir ces mêmes concurrents comme des partenaires sur un marché qui se mondialise. Les intervenants rencontrés en entrevues ont été unanimes à souligner la grande difficulté de changer les mentalités et de créer cet esprit de collaboration pourtant nécessaire à la survie de l'industrie. À ce sujet, Allaire et Firsirotu (1993) parlent de « la myopie des dirigeants » qui considèrent que l'augmentation de leurs parts de marché passe nécessairement par la diminution de celles de leurs concur- 
rents immédiats. Le manque de confiance entre partenaires freine également une certaine collaboration intra-industrie. Les entreprises filleules non-participantes hésitent, pour leur part, à laisser pénétrer dans l'enceinte de leurs murs leurs donneurs d'ordres de crainte que ces derniers ne découvrent certaines des difficultés ou des faiblesses de leur processus de production. Cet état de fait reflète le climat de méfiance qui, trop souvent, caractérise les relations entre sous-traitants et donneurs d'ordres. Bourgault (1996) a pourtant démontré que les PME très performantes dans le secteur de l'aérospatiale misent sur une étroite collaboration avec leurs donneurs d'ordres, collaboration qui favorise le transfert de compétences.

Certaines organisations approchées pour parrainer une PME ont invoqué un manque de temps ou de ressources pour motiver leur refus. La collaboration exige un climat de confiance mutuelle et d'entraide (Ring Smith et Van de Ven, 1994) encore peu présent dans les entreprises et chez leurs dirigeants. Les grandes entreprises souffrent donc également d'une certaine myopie qui les empêche de percevoir les avantages qu'elles retireront à s'impliquer dans une relation durable avec leurs sous-traitants. Une étude réalisée par Hart et Saunders (1997) démontre que des relations basées sur le pouvoir mènent à une adoption plus restreinte de l'échange de données informatisé (ÉDI) alors que des relations de confiance facilitent et accélèrent cette adoption. La grande entreprise désireuse d'améliorer la qualité de ses produits et de ses composants ne peut donc pas négliger le choix de ses soustraitants et doit s'impliquer activement pour les soutenir dans leurs efforts d'amélioration. Ghobadian et Gallear (1996) confirment en effet que l'adoption d'un programme de qualité par un sous-traitant affecte positivement la compétitivité de l'entreprise cliente.

Que dire des entreprises qui ont accepté de relever le défi de la collaboration et d'adhérer au projet de parrainage pour l'implantation des normes ISO 9000 ? Les résultats de cette étude ont révélé que ces entreprises jouissent d'une culture organique par opposition à une industrie de type mécanique et de mécanismes de décision de type participatifs, du moins pour les entreprises filleules. En cela, elles privilégient la coopération plutôt que le contrôle et favorisent l'habilitation et la responsabilisation de leurs employés. Cependant, ni le degré d'innovation de leur stratégie manufacturière, ni l'avant-gardisme de leurs mécanismes de gestion des ressources humaines ou de formation ne permettent de les différencier des entreprises non participantes. Un des principaux intervenants sur ce dossier, rattaché au MISCT, expliquait que, selon lui, à ce stade-ci du projet, nous sommes en présence d'individus convaincus du bien-fondé de la collaboration mais non d'entreprises collaboratrices. Une première expérience concluante contribuera, si c'est le cas, à véritablement «changer les esprits dans la relation entre les sous-traitants et les donneurs d'ordres » (Julien, 1994, p. 55) et à créer cette vision à long terme et cette synergie créatrice dont l'industrie a grand besoin. 


\section{Bibliographie}

ACS, Z.J. et D.B. AUdRETSCH (1989), « Job creation and firm size in the U.S. and West Germany ", International Small Business Journal, vol. 7, $\mathrm{n}^{\circ}$ 4, p. 9-22.

Allaire, Y. et M.E. Firsirotu (1993), L'entreprise stratégie: Penser la stratégie, Montréal, Gaëtan Morin Éditeur.

BIRLEY, S. et P. WESTHEAD (1990), « Growth and performance contrasts between types of small firms », Strategic Management Journal, vol. 17, $\mathrm{n}^{\circ}$ 7, p. 535-557.

Bourgault, M. (1996), Compétences des entreprises et sous-traitance nord-américaines: le cas de l'industrie aérospatiale, Thèse, École polytechnique de Montréal.

BRowning, L.D., J.M. BEYER et J.C. SHETLER (1995), «Building cooperation in a competitive industry : sematech and the semi-conductor industry ", Academy of Management Journal, vol. 38, no 1, p. 113-151.

Burgers, W.P., C.W. HiLl et K.W. CHAN (1993), «A theory of global strategic alliances : the case of the global auto industry », Strategic Management Journal, vol. 14, janvier, p. 419-432.

COVIN, J.G. et D.P. SLEVIN (1990), «New venture strategic posture, structure and performance : an industry life cycle analysis », Journal of Business Venturing, vol. 5, $\mathrm{n}^{\mathrm{o}} 2$, p. $123-135$.

ETTLIE, J.E. (1983), «Organizational policy and innovation among suppliers to the food processing sector », Academy of Management Journal, vol. 26, n 1, p. 27-44.

ETTLIE, J.E. et J.D. PENNER-HAHN (1990), «Focus, modernization, and manufacturing technology policy », dans comptes rendus de Joint Industry University Conference on Manufacturing Strategy, J.E. Ettlie, M.C. Burstein et A. Fiegenbaum (éd.), Manufacturing Strategy - The Research Agenda for the Next Decade, Boston, Kluwer Academic Publishers, p. 153-164.

Gagné, P. et M. LefÈvre (1993), L'atlas industriel du Québec, Montréal, Publi-Relais.

GHobadian, A. et D.N. GalleAR (1996), « Total quality management in SMEs », Omega, International Journal of Management Science, vol. 24, $\mathrm{n}^{0}$ 1, p. 83-106.

GouvernEMENT du QUÉBEC (1995), Ma force, c'est la force de mon réseau, Québec, Gouvernement du Québec.

GRAY, B. et D. WOOD (1991), «Collaborative alliances : moving from practice to theory », Journal of Applied Behavioral Science, vol. 27, nº 1, p. 3-22.

GuilhoN, B. (1993), «Les relations entre constructeurs et fournisseurs : l'exemple de l'industrie automobile», Revue Internationale PME, vol. 6, n 1, p. 87-99.

HART, P. et C. SAUNDERS (1997), «Power and trust : critical factors in the adoption and use of electronic data interchange », Organization Science, vol. 8, $\mathrm{n}^{\circ} 1$, p. 23-42.

HoRNSBY, J. et D. KURATKo (1990), «Human resource management in small business: critical issues for the 1990's », Journal of Management, vol. 18, $\mathrm{n}^{\circ} 1$, p. 45-57.

JULIEN, P.-A. (1994), «L'entreprise partagée : contraintes et avantages », Gestion, décembre, p. $48-58$. 
KANTER, R.M. (1983), The Change Masters - Innovation Corporation, New York, Simon \& Schusters Inc.

KUMAR, N. (1996), «The power of trust in manufacturer-retailer relationships », Harvard Business Review, novembre-décembre, p. 92-106.

LEFEBVRe, É., L.A. LEFEBVRE et L. PrÉFOnTAINE (1993), « Innovative efforts as predictors of quality, diversity and cost dimensions of manufacturing strategy », dans Productivity \& Quality Management Frontiers - IV, D.J. Sumanth, J.A. Edosomwan, R. Poupart et D.S. Sink (éd.), Norcross Georgia, Industrial Engineering and Management Press, p. 526-534.

MASSE, J.-M. (1992), Le changement planifié et la gestion de la qualité, Sillery (Québec), Presses de l'Université du Québec, p. 317-361.

Mody, A. (1993), «Learning through alliances », Journal of Economic Behavior and Organization, vol. 20, $\mathrm{n}^{\circ} 2$, p. 151-170.

NARO, G. (1990), «Les PME face à la gestion de leurs effectifs : comment adapter les ressources humaines aux impératifs stratégiques? », Revue Internationale PME, vol. $3, \mathrm{n}^{\circ} 1$, p. $57-74$.

NeBENHAUS, D. (1990), «Les structures d'influence du personnel sur les décisions : enquête auprès de $\mathrm{PME} »$, Revue Internationale $P M E$, vol. $3, \mathrm{n}^{\circ}$ 1, p. 89-104.

Poulin, D., B. MonTreuIL et S. GAUVIN (1994), L'entreprise réseau : Bâtir aujourd'hui l'organisation de demain, Montréal (Québec), Publi-Relais.

Préfontaine, L., L.A. LefEBVRE et É. LefEBVRE (1995), «Organizational capabilities and R\&D intensity: is there a link ?", Technology Management, vol. 95, $\mathrm{n}^{\circ} 2$, p. 31-40.

RING SMITH, P. et A.H. VAN DE VEN (1994), «Developmental processes of cooperative interorganizational relationships ", Academy of Management Review, vol. 19, no 1 , p. $90-118$.

Rogers, E.M. (1983), Diffusion of Innovations, New York, The Free Press.

Rosanvallon, A. (1990), «Les politiques de formation dans les PME-PMI françaises: l'émergence de pratiques novatrices », Revue Internationale PME, vol. $3, \mathrm{n}^{\circ} 1$, p. 75-87.

ROTHWELL, R. (1991), «External networking and innovation in small and medium-sized manufacturing firms in Europe», Technovation, vol. 11, n 2, p. 93-112.

SHARMAN, M.P., B. GRAY et A. YAN (1991), «The context of interorganizational collaboration in the garment industry : an institutional perspective », Journal of Applied Behavioral Science, vol. 27, $\mathrm{n}^{\circ} 2$, p. 81-208.

Société québécoise de développement de la main-d'œuvre (SQDM) (1994), «Le Parrainage ». Guide d'élaboration d'un projet de parrainage, Montréal, Direction des services aux entreprises.

UZAN, O. (1990), «La gestion des ressources humaines en PME: Quelques repères pour la recherche », Revue Internationale PME, vol. 3, no 1, p. 106-16. 
WeSTLEY, F. et H. VREDENBURG (1991), « Strategic bridging : the collaboration between environmentalists and business in the marketing of green products », Journal of Applied Behavioral Science, vol. 27, $\mathrm{n}^{0} 1$, p. 91-115.

Williamson, O.E. (1981), «The modern corporation : origins, evolution attributes », Journal of Economics Literature, tome XIX, p. 1537-1568. 